\title{
Predictability of Inverse Impact Force Location as Affected by Measurement Noise
}

\author{
Abdelali El-Bakari, ${ }^{1}$ Abdellatif Khamlichi, ${ }^{2,3}$ Rachid Dkiouak, ${ }^{1}$ \\ Ali Limam, ${ }^{4}$ and Eric Jacquelin ${ }^{5,6}$ \\ ${ }^{1}$ Civil Engineering and Mechanics Laboratory, Abdelmalek Essaâdi University, 91001 Tangier, Morocco \\ ${ }^{2}$ Communications Systems and Detection Laboratory, Abdelmalek Essaâdi University, 93002 Tetouan, Morocco \\ ${ }^{3}$ Department of Physics, Faculty of Sciences at Tetouan, P.O. Box 2121, M'Hannech II, 93002 Tetouan, Morocco \\ ${ }^{4}$ Civil and Environmental Engineering Laboratory, Institute of Applied Sciences at Lyon, 20 Albert Einstein avenue, \\ 69621 Villeurbanne Cedex, France \\ ${ }^{5}$ University of Lyon, 69622 Lyon, France \\ ${ }^{6}$ IFSTTAR, LBMC, UMR-T9406, Université Lyon 1, Villeurbanne, France
}

Correspondence should be addressed to Abdellatif Khamlichi; khamlichi7@yahoo.es

Received 17 June 2013; Accepted 4 September 2013

Academic Editors: J. L. C. Fonseca, T. Matsumoto, and M. Saitou

Copyright (C) 2013 Abdelali El-Bakari et al. This is an open access article distributed under the Creative Commons Attribution License, which permits unrestricted use, distribution, and reproduction in any medium, provided the original work is properly cited.

\begin{abstract}
The impact force localization inverse problem is considered through a nonlinear optimization procedure. The objective function is derived in the particular case of elastic structures for which Maxwell-Betti theorem holds. Additional geometric constraints were introduced in order to stabilize optimum search. The solution of the constrained non linear mathematical problem was performed by means of two outstanding evolutionary algorithms that include Genetic Algorithm and Particle Swarm Optimization. Focus was done on the robustness aspect of force impact localization predictability when an additive white noise is assumed to perturbed strain measurement. It was found that the Genetic Algorithm fails to track the exact solution independently from the noise level as an error was systematically present in the solution. On the other hand, the Particle Swarm Optimisation based algorithm performed very well even for noise levels as high as $2 \%$ of the measured strain signal.
\end{abstract}

\section{Introduction}

Identification of impact force location for impact events occurring on elastic structures can be performed by various methods that were proposed in the literature [1-3]. To review briefly some of the important contributions in this filed, Martin and Doyle [4] have described how to find the location of an impact force using dynamic response measurements. They proposed a solution procedure using the spectral element method with a stochastic iterative search. Experimentally measured acceleration responses from two frame structures were used to achieve force localization by minimizing a fitness function. A Genetic Algorithm was used to guess iteratively the minimum through monitoring the actual error associated to a given sampling generation. The process enabled to discriminate between good and bad guesses and gave at convergence the correct impact location. An alternative technique which employs the arrival time of each frequency component of a pulse detected by means of wavelet transform was proposed by Inoue et al. [5]. But this approach suffers from the lack of accuracy in measurement of small arrival times of signals. Yen and $\mathrm{Wu}[6,7]$ have used multiple strain responses along with a mutuality relationship based on Green's functions and measured strains to achieve identification of force location on two-dimensional plate-like structures. Choi and Chang [8] minimized the error between measured strain responses in PZT sensors and numerically evaluated impact force locations. Shin [9] proposed a technique for identifying the force location using modal displacements and transient signal measured by accelerometers. 
As mentioned by Doyle [10], the implicit character of force localization requires considering the inverse problem of force identification as being rather associated to two decoupled subproblems: localization of impact force and reconstruction of the time force signal. The reason for this is that the inverse problem of force identification cannot be handled by just adding the force point coordinates as extra unknown parameters to the discrete vector of time force signal values. These positions intervene in fact as implicit parameters, in contrast with the force history values which are explicit. Iterations are then required for solution of force positions unknowns in order to determine them at first. Treating all force history values to be implicit parameters is not a good choice since it would increase dramatically the number of unknowns and would consequently penalize the computational cost. When the force localization is obtained through the solution of a nonlinear mathematical program, the force time signal reconstruction can then be performed by means of a regularization deconvolution technique.

In this work, separation of the localization and reconstruction phases of the impact force inverse problem is adopted. Elastic structures subjected to nonpunctual impacts for which the resulting force field can be assumed to be uniformaly distributed over a finite domain of the structure are considered. The localization problem is solved by using minimization procedures that are of evolutionary type. Two methods are examined in the following: Genetic Algorithm (GA) based strategy [11] and the Particle Swarm Optimization (PSO) approach $[12,13]$. Focus will be done on the particular role related to noise affecting strain measurement as to the resulting perturbations that produce and which may impede these algorithms to converge towards the exact problem solution.

The localization problem is derived straightforwardly from the reciprocity Maxwell-Betti theorem which is valid for any elastic structure. However, as the direct expression of the fitness function formed by this theorem is ill conditioned, because it admits a lot of trivial meaningless solutions which are associated to the fixed boundary conditions, the fitness function is modified [7]. The introduced modification transforms the nonlinear mathematical program to a unique solution problem and removes the trivial parasitic solutions which complicate optimization process convergence to the real solution. Some extra constraints are also introduced in order to guide the exploration of the optimal solution; these are associated to the geometric pieces of evidence that describe the domain containing the tracked unknowns.

The aim is to examine the predictability of impact location in this situation where noise is present in observation measurement. This means determining the amount of noise that can be tolerated and also selecting between the two proposed algorithms the most suited one that can be used to conduct solution of force localization problem with sufficient accuracy and minimal error.

\section{Materials and Methods}

2.1. Direct Problem Formulation. Although the problem can be stated for any elastic structural system, a simplified model

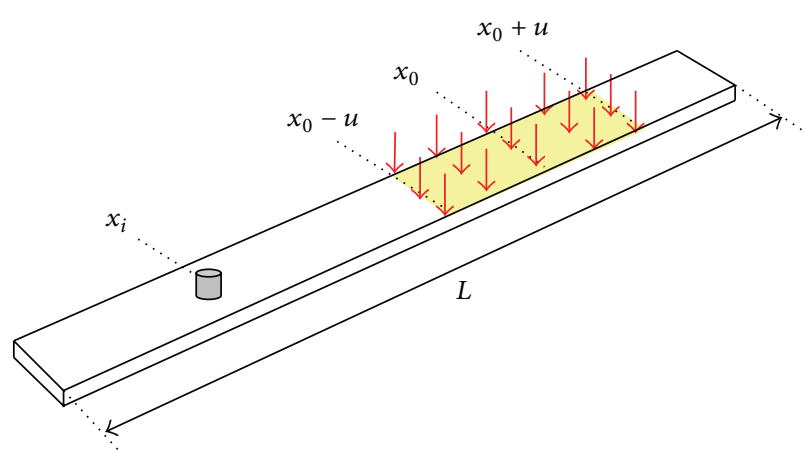

Figure 1: An elastic beam with a uniform rectangular crosssection and loaded with a distributed uniform pressure; four sensors positions are indicated.

having the form of a beam with a rectangular uniform section is considered, Figure 1 . The beam is assumed to be simply supported on both ends. It has length $L$, width $b$, and height $e$. It is assumed to be made from a homogeneous and isotropic elastic material with Young's modulus $E$ and density $\rho$. The applied force modelling impact is assumed to result from a uniformly distributed pressure, $p$, which is applied on a rectangular patch as shown in Figure 1. The pressure rectangle is assumed to be centred on $x_{0}$ and of length $2 u$.

The differential equation for transverse vibrations of a beam writes

$$
\rho S \frac{\partial^{2} y(x, t)}{\partial t^{2}}+c \frac{\partial y(x, t)}{\partial t}+E I \frac{\partial^{4} y(x, t)}{\partial x^{4}}=p(x, t),
$$

where $y$ is the transverse displacement, $x$ is the axial position, $t$ is the time, $S$ is the cross-section area, $\rho$ is the density, $E$ is Young's modulus, $I$ is the moment of inertia, $c$ is the viscous damping coefficient, and $p(x, t)$ is the applied pressure over $\left[x_{0}-u, x_{0}+u\right]$.

The considered boundary conditions are as follows:

$$
\begin{gathered}
y(0, t)=y(L, t)=0, \\
\frac{\partial^{2} y}{\partial t^{2}}(0, t)=\frac{\partial^{2} y}{\partial t^{2}}(L, t)=0 .
\end{gathered}
$$

Using modal superposition and Duhamel's integral, the transient dynamic solution of the impacted beam in terms of axial strain $\varepsilon$ at location $x_{i}$ is given by the following convolution problem:

$$
\varepsilon\left(x_{i}, t, x_{0}, u\right)=\int_{0}^{t}\left(\int_{x_{0}-u}^{x_{0}+u} g\left(x_{i}, \zeta, t-\tau\right) p(\zeta, \tau) d \zeta\right) d \tau,
$$

where $g\left(x_{i}, \zeta, t-\tau\right)$ is the time response function between point $\zeta$ and the sensor location $x_{i}$.

Time discretisation of (3) under the assumption that the pressure $p(\zeta, \tau)$ is uniform yields the following linear system:

$$
Y_{i}=G_{i}\left(x_{0}, u\right) P,
$$


where $Y_{i}$ designates the vector containing the discrete values of axial strain at point location indicated by index $i, P$ is the vector containing discrete time values of the applied uniform pressure, and $G_{i}\left(x_{0}, u\right)$ is the Toeplitz-like matrix.

This last has the following form:

$$
G=\left[\begin{array}{cccc}
g(1) & 0 & \cdots & 0 \\
g(2) & g(1) & \ddots & \vdots \\
\vdots & \vdots & \ddots & 0 \\
g(N) & g(N-1) & \cdots & g(1)
\end{array}\right]
$$

where $N$ is the number of time steps.

An explicit expression can be obtained for matrix $G$. Let us denote by $\Delta t$ the time step used in discretization and by $M$ the order of modal truncation; the impulse response function giving the deformation of the top fiber for a section having abscissa $x_{i}$ is given by

$$
\begin{gathered}
G_{k j}\left(x_{i}, x_{0}, u\right) \\
=\frac{4 \pi}{\rho S L^{2}} \sum_{m=1}^{M} \sin \left(\frac{m \pi x_{0}}{L}\right) \sin \left(\frac{m \pi u}{L}\right) \sin \left(\frac{m \pi x_{i}}{L}\right) \\
\times g\left(\omega_{m}, \xi_{m},(j-k) \Delta T\right),
\end{gathered}
$$

with $g\left(\omega_{m}, \xi_{m},(j-k) \Delta T\right)=\left(m \exp \left(-\xi_{m} \omega_{m}(j-\right.\right.$ $\left.k) \Delta T) / \omega_{m} \sqrt{1-\xi_{m}^{2}}\right) \sin \left(\omega_{m} \sqrt{1-\xi_{m}^{2}}(j-k) \Delta T\right)$ if $j \geq k$, and $g\left(\omega_{m}, \xi_{m},(j-k) \Delta T\right)=0$ if $j<k$, where $\omega_{m}=\left(m^{2} \pi^{2} / L^{2}\right) \sqrt{E I / \rho S}$ and $\xi_{m}$ are, respectively, the circular eigenfrequency and damping ratio for a given eigenmode $m$.

2.2. Localization Problem as Perturbed by the Presence of Measurement Noise. The problem of finding the impact location for the beam considered in the present study consists in identifying the impact patch centre position $x_{0}$ and parameter $u$ defining the extent of the impacted zone. Using (4) to (6), the responses measured by strain sensors placed at points having the abscissa $x_{i}$ and $x_{j}$ can be expressed, respectively, under the following form:

$$
\begin{gathered}
Y_{i}=G_{i}\left(x_{0}, u\right) P, \\
Y_{j}=G_{j}\left(x_{0}, u\right) P .
\end{gathered}
$$

Equation (7) can be used to prove the following important commutativity property which does not contain the pressure vector $P$ :

$$
G_{j}\left(x_{0}, u\right) Y_{i}-G_{i}\left(x_{0}, u\right) Y_{j}=0 .
$$

In this way, $\left(x_{0}, u\right)$ appears to be the solution of the following equation:

$$
\left\|G_{j}(s, v) Y_{i}-G_{i}(s, v) Y_{j}\right\|^{2}=0,
$$

where $\|\cdot\|$ is the Euclidian norm.

There are a lot of other trivial solutions for (9) for which the factor $\sin (m \pi s / L) \sin (m \pi v / L)$ appearing in the second hand side of (6) vanishes for all values of $m \in[1, M]$. These are associated to the boundaries of the domain containing $\left(x_{0}, u\right)$; that is, $\{s=0 ; s=L ; v=0 ; v=L\}$. To get the exact solution of impact location, the parasitic solutions should be withdrawn from (9).

Denoting by $N_{s}$ the number of sensors used, the fitness function to be minimized in order to find the impact zone parameters $\left(x_{0}, u\right)$ is proposed under the following form:

$$
\begin{aligned}
& \left(x_{0}, u\right) \\
& =\underset{(s, v)}{\operatorname{Arg} \min }\left\{\phi(s, v)=\sum_{i=1}^{N_{s}} \sum_{\substack{j=1 \\
j \neq i}}^{N_{s}} \frac{1}{\alpha_{i j}}\left\|G_{j}(s, v) Y_{i}-G_{i}(s, v) Y_{j}\right\|^{2}\right\},
\end{aligned}
$$

with $\alpha_{i j}=\left\|G_{i}(s, v)\right\|^{2}+\left\|G_{j}(s, v)\right\|^{2}$ defining the weighting coefficients that are introduced in order to remove the parasitic solutions, as they vanish also for $\{s=0 ; s=L ; v=$ $0 ; v=L\}$.

In the presence of measurement noise, the perturbed fitness function takes the following form:

$$
\phi(s, v)=\sum_{i=1}^{N_{s}} \sum_{\substack{j=1 \\ j \neq i}}^{N_{s}} \frac{1}{\alpha_{i j}}\left\|G_{j}(s, v) \tilde{Y}_{i}-G_{i}(s, v) \tilde{Y}_{j}\right\|^{2}
$$

with

$$
\begin{gathered}
\tilde{Y}_{i}=\left(1+v_{i} z_{k}\right)_{i}\left(Y_{k}\right)_{i}, \\
\tilde{Y}_{j}=\left(1+v_{j} z_{k}\right)_{j}\left(Y_{k}\right)_{j},
\end{gathered}
$$

where $z_{k}$ is a random number belonging to the interval $[-1,1]$ and $v_{i}$ and $v_{j}$, respectively designate the noise level present in measurement delivered by sensor $i$ and sensor $j$, respectively.

To stabilize the minimization procedure and obtain the unique physical solution, the unconstrained mathematical program defined by (10) is constrained by adding the following geometrical bounds conditions:

$$
\begin{gathered}
\frac{L}{40} \leq x_{0} \leq L-\frac{L}{40}, \\
\frac{L}{40} \leq u \leq \frac{L}{2} .
\end{gathered}
$$

To solve the mathematical program defined by (6) and (10)(13), the PSO algorithm and the GA are considered. Their performance will then be assessed as function of the random noise level present in strain measurement.

2.3. PSO Algorithm. A PSO based method was proposed initially by Eberhart and Kennedy $[12,13]$. This approach has gained since then considerable interest as being one of the most promising optimization methods that is able to provide high speed and high accuracy. PSO mimics the social behavior that a population of individuals adapts to its environment by returning to promising regions that were previously discovered [14]. This adaptation to the environment is 
a stochastic process that depends on both the memory of each individual, called particle, and the knowledge gained by the population, called swarm.

In the simplest numerical implementation of this method, each particle is characterised by four attributes: the position vector in the search space, the velocity vector, the best position achieved in its track, and the best position achieved by the swarm. The process steps can be outlined as follows.

Step 1. Generate the initial swarm involving $N$ given particles placed at random.

Step 2. Calculate the new velocity vector of each particle, based on its actual attributes.

Step 3. Calculate the new position of each particle from the current position and its new velocity vector.

Step 4. If the termination condition is satisfied, stop. Otherwise, go to Step 2.

To be more specific, the new velocity vector of the $i$ th particle at time $t+1$, denoted $v_{i}^{t+1}$, is calculated according to the following Shi and Eberhart [15] formula:

$$
v_{i}^{t+1}=\omega^{t} v_{i}^{t}+c_{1} R_{1}^{t}\left(p_{i}^{t}-x_{i}^{t}\right)+c_{2} R_{2}^{t}\left(p_{g}^{t}-x_{i}^{t}\right) .
$$

In (14), $R_{1}^{t}$ and $R_{2}^{t}$ are random numbers between 0 and 1 , $p_{i}^{t}$ is the best position of the ith particle in its track, and $p_{g}^{t}$ is the best position of the swarm. There are three problem dependent parameters that fix performance of this algorithm, namely, the inertia of the particle $\omega^{t}$ and the two trust parameters $c_{1}$ and $c_{2}$.

The new position of the $i$ th particle at time $t$, denoted $x_{i}^{t+1}$, is then calculated as follows:

$$
x_{i}^{t+1}=x_{i}^{t}+v_{i}^{t+1}
$$

where $x_{i}^{t}$ is the current position of the $i$ th particle at time $t$. The $i$ th particle actual position enables to determine the best position in its track $p_{i}^{t}$. When considering all the particles, the global best position of the swarm $p_{g}^{t}$ is then obtained.

PSO algorithm works such that particles concentrate on the best search position of the swarm. They cannot easily escape from the local optimal solution since the search direction vector $v_{i}^{t+1}$ calculated by (14) always includes the direction vector to the best search position of the swarm. This shows the major feature of PSO algorithm as being a robust process of continuous enhancement for optimum search.

In the presence of constraints, a particle move should be restricted in order to remain in the feasible solution space by examining the given constraints. A modified PSO version was introduced for constrained problems in order to manage this situation [16].

2.4. Genetic Algorithm. Genetic Algorithm (GA) was firstly introduced by Holland [11]. It is a probabilistic optimization method that is able to achieve global search by mimicking natural biological evolution. GA operates on a population of individuals called the set of potential solutions. Each individual is represented by an encoded string (chromosome) that contains the decision variables (genes). Traditionally, GA uses binary strings as chromosome representation.

The GA has an iterative procedure structure that comprises generally the following five main steps.

Step 1. Creating an initial population $\left(G_{0}\right)$.

Step 2. Evaluation of the performance of each individual or chromosome $\left(c_{k}\right)$ of the population, by means of a fitness function to be maximized.

Step 3. Selection of individuals for the reproduction of a new population.

Step 4. Application of genetic operators: Crossover and Mutation.

Step 5. Iteration of Steps 2 to 4 until a termination criterion is fulfilled.

In the localization problem considered in this work, the candidate solution is the centre position $s_{0}$ and the extent of the impact zone $u$. These variables are then coded in a chromosome using a binary coding scheme.

To start the algorithm, an initial population of individuals (chromosomes) is defined. The GA is configured, so that it creates a fixed number of initial individuals at random from the whole feasible solution space. An important parameter in initialization is the population size. In general, the population size affects both the ultimate performance and the efficiency of GA and should be determined in a case by case study.

\section{Results and Discussion}

A pinned-pinned beam having the following material and geometric properties is considered: $E=7.06 \times 10^{10} \mathrm{~Pa} ; L=$ $0.5 \mathrm{~m} ; b=e=5 \times 10^{-3} \mathrm{~m} ; \rho=2660 \mathrm{~kg} \cdot \mathrm{m}^{-3} ; \xi_{m}=2 \%$; $x_{0}=0.417 \mathrm{~m} ; u=0.0417 \mathrm{~m}$. The time interval considered has the duration $T_{c}=1 \mathrm{~s}$. The beam is assumed to be subjected to a half sine pulse pressure having the shape and spectral content depicted in Figure 2. The maximum pressure is taken to be $10^{5} \mathrm{~Pa}$.

The first five modes were retained. Their frequencies are given by $f_{1}=46.72 \mathrm{~Hz}, f_{2}=186.9 \mathrm{~Hz}, f_{3}=420.5 \mathrm{~Hz}, f_{4}=$ $747.5 \mathrm{~Hz}$, and $f_{5}=1168 \mathrm{~Hz}$. The time step used was $\Delta t=$ $3.4246 \times 10^{-4} \mathrm{~s}$ which satisfies largely Shannon condition with regards to $f_{5}$.

Four gauge strain sensors were used in this study. Their labeling and positions are indicated in Table 1. Previous studies conducted by means of PSO algorithm have shown that, while considering the noise free problem, localization needs at least three sensors to be achieved with adequate accuracy. Here, a fourth sensor has been added in order to enhance the performance of the localization inverse problem in the presence of measurement noise. 


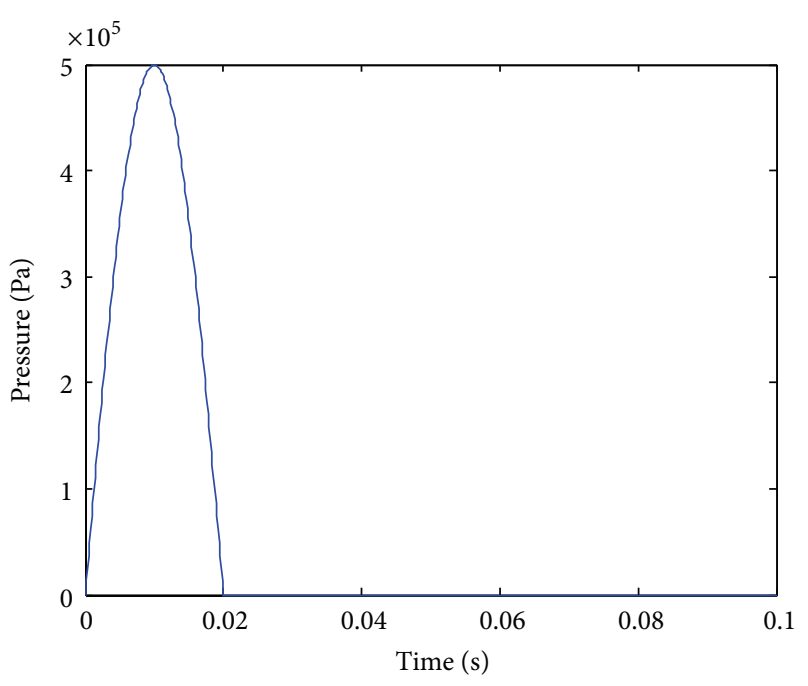

(a)

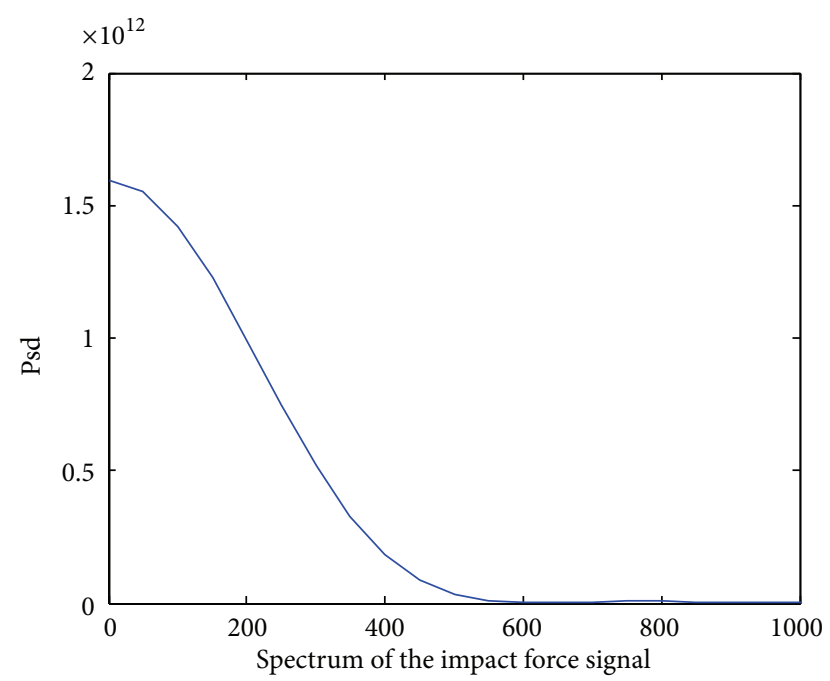

(b)

FIGURE 2: Characteristics of the impulse impact pressure; (a) time history and (b) frequency content.

TABLE 1: Positions of the gauge sensors considered for the measurement of axial strain at the upper beam fiber.

\begin{tabular}{lc}
\hline Sensor & Position \\
\hline$\# 1$ & $5 L / 24$ \\
$\# 2$ & $L / 3$ \\
$\# 3$ & $L / 2$ \\
$\# 4$ & $2 L / 3$ \\
\hline
\end{tabular}

Three noise levels were considered in order to test robustness of both PSO and GA based algorithms. They correspond to the following.

Case 1. Noise free case where noise amplitude is set to the value $v_{i}=0 \%$ for the four sensors.

Case 2. Noise level is fixed at $v_{i}=2 \%$ for the four sensors.
Case 3. Noise level is fixed at $v_{i}=5 \%$ for the four sensors.

The parameters used for GA were as follows:

(i) stopping test: $10^{-6}$;

(ii) population size: 100;

(iii) probability of intersection: 1 ;

(iv) probability of mutation: 0.05 ;

(v) maximum number of generation: 200.

For GA algorithm the first values of the unknown parameters impact centre and extent were initialized with $x_{0}=u=$ $1.25 \times 10^{-2}$.

For PSO algorithm, the following stability parameters were used: $w=0.4 ; c_{1}=1.25 ; c_{2}=0.5$; size population: 100 .

Figure 3 gives evolution of impact location characteristics as a function of iterations in case of noiseless problem. One can see the difference existing between these two algorithms; PSO algorithm gives the exact solution of the impact location. On the opposite, GA has not converged to the right solution.

Figure 4 gives evolution of impact location characteristics as function of iterations in case of noisy measurement conditions with a noise level $\nu_{i}=2 \%$. One can observe that GA has not converged at all, as the calculated solution is too far from the exact solution. The relative error reached $92 \%$ for the impact zone extent. Meanwhile, PSO based algorithm has continued to give the exact solution with only a moderate error, representing $13 \%$ of the impact zone extent.

Figure 5 gives the evolution of impact location characteristics as function of iterations in case of noise level value given by $v_{i}=5 \%$. Unexpectedly, GA is better in this condition than for noise level $v_{i}=2 \%$ as the relative error for impact zone extent has decreased from $92 \%$ to $68 \%$. Moreover, one can see that both GA and PSO algorithms fail to give the exact solution as the error on the impact zone extent is too large, $61 \%$ and $68 \%$, respectively.

Tables 2 and 3 recall the obtained relative error as the function of the algorithm used and noise level. One can see that GA fails to predict the exact solution, with a behavior that is not monotonous, while $\mathrm{PSO}$ based algorithm performs well for small measurement noise levels not exceeding $2 \%$. The performance decreases after that when identifying the impact zone extent, while it remains sufficiently good for the impact zone centre as the relative error is smaller than $5 \%$.

To emphasize the irregular behavior of GA, a comparison has been made between the results of two runs of GA. Figure 6 shows a comparison between the obtained evolutions. They are not the same because of the stochastic nature of GA. GA is found thus to be inappropriate for the inverse force location problem as it is too sensitive to noise level and its repeatability is too poor.

On the opposite, PSO algorithm runs always in the same manner with perfect repeatability.

\section{Conclusion}

Based on the separation approach that decouples force location from force signal reconstruction in an inverse 


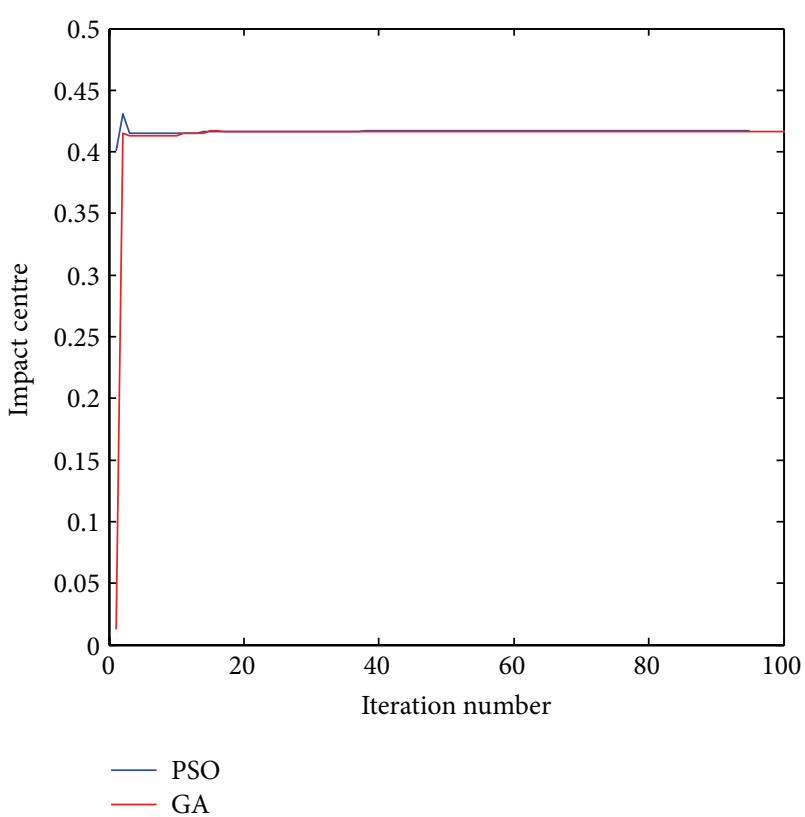

(a)

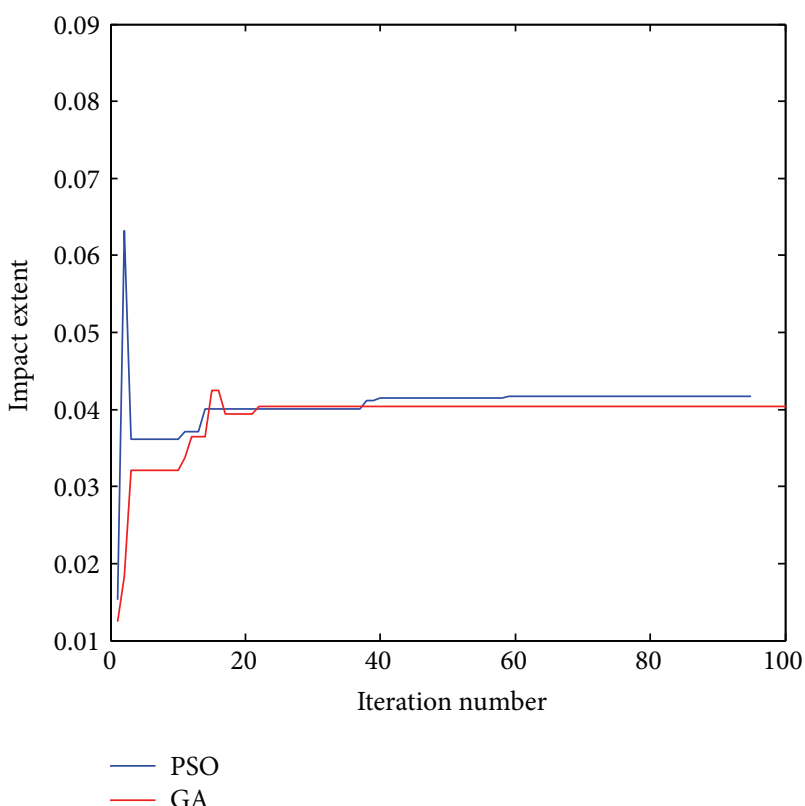

(b)

FIGURE 3: Noise free case; evolution of impact zone characteristics. (a) Impact centre and (b) impact zone extent.

TABLE 2: Relative error affecting impact zone extent $u$ as compared with the exact solution.

\begin{tabular}{lccc}
\hline & $0 \%$ & $2 \%$ & $5 \%$ \\
\hline PSO & 0 & $1.271 e-001$ & $6.163 e-001$ \\
GA & $-3.113 e-002$ & $9.198 e-001$ & $6.800 e-001$ \\
\hline
\end{tabular}

impact problem occurring on an elastic beam, robustness of a particular localization procedure was analyzed. This uses a modified fitness function derived from MaxwellBetti theorem by applying some filtering coefficients that

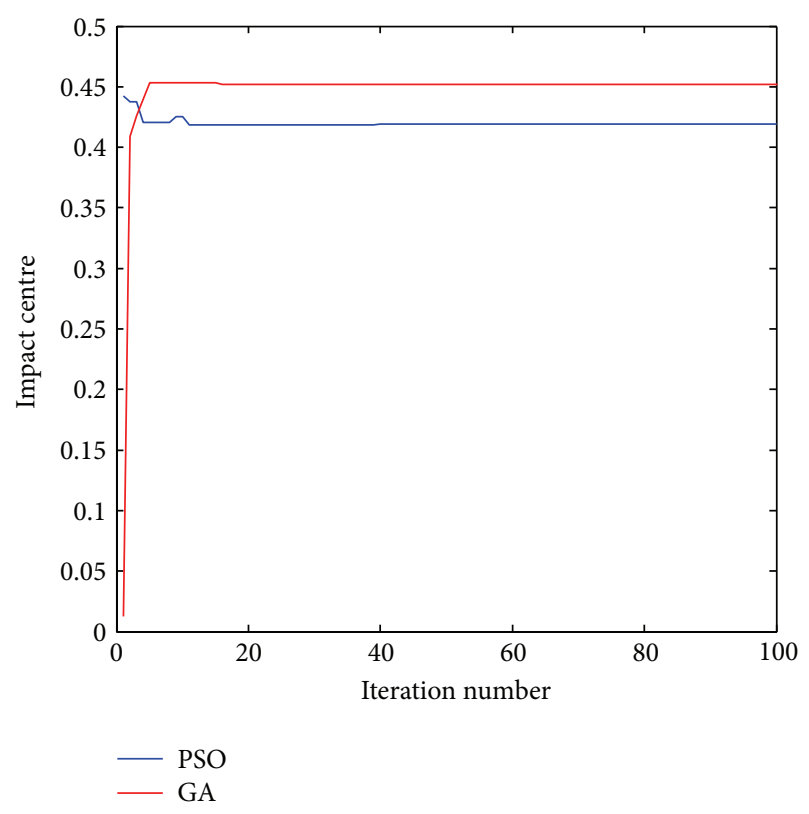

(a)

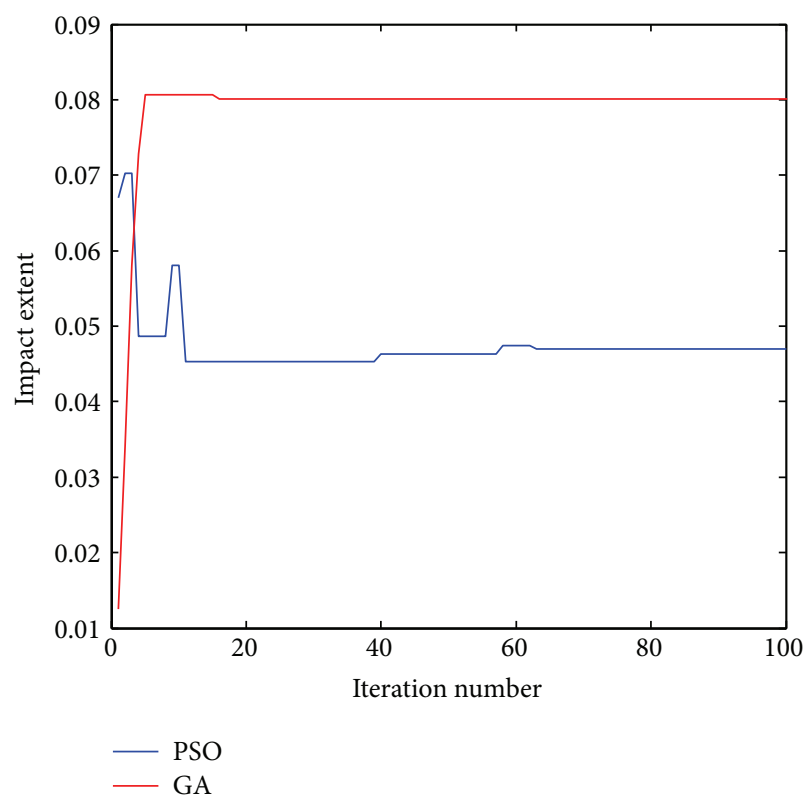

(b)

FIgURE 4: Noise level $\nu_{i}=2 \%$; evolution of impact zone characteristics. (a) Impact centre and (b) impact zone extent.

TABLE 3: Relative error affecting impact zone centre $x_{0}$ as compared with the exact solution.

\begin{tabular}{lccc}
\hline & $0 \%$ & $2 \%$ & $5 \%$ \\
\hline PSO & 0 & $5.217 e-003$ & $3.765 e-002$ \\
GA & $-1.378 e-003$ & $8.294 e-002$ & $4.428 e-002$ \\
\hline
\end{tabular}

enable to remove parasitic solutions. Solution of the obtained constrained nonlinear mathematical program that provides the impact zone location was performed by GA and PSO based algorithms. Predictability of force location was studied 


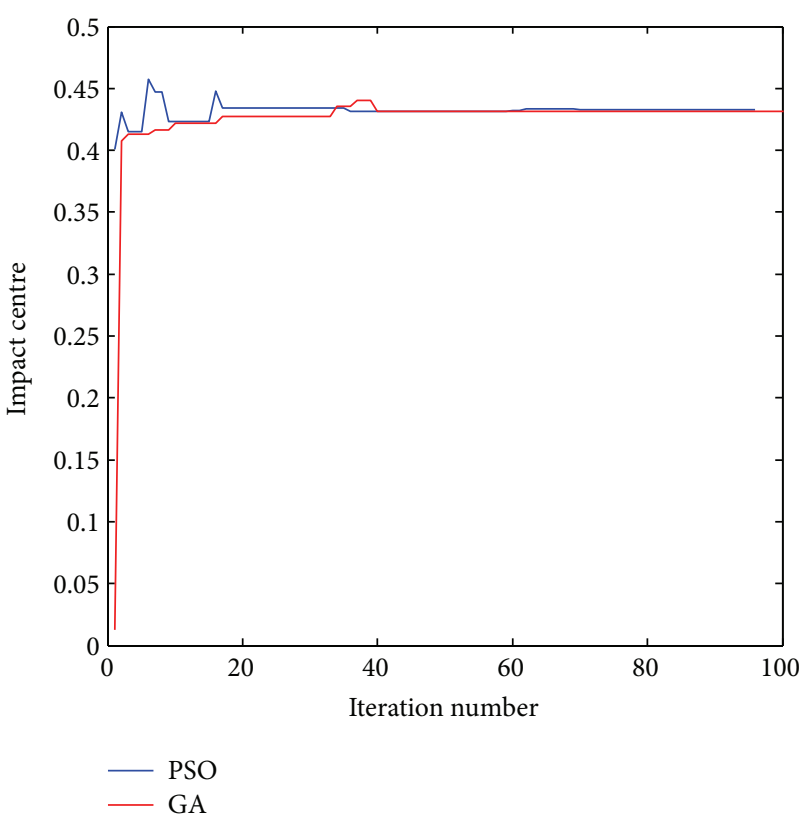

(a)

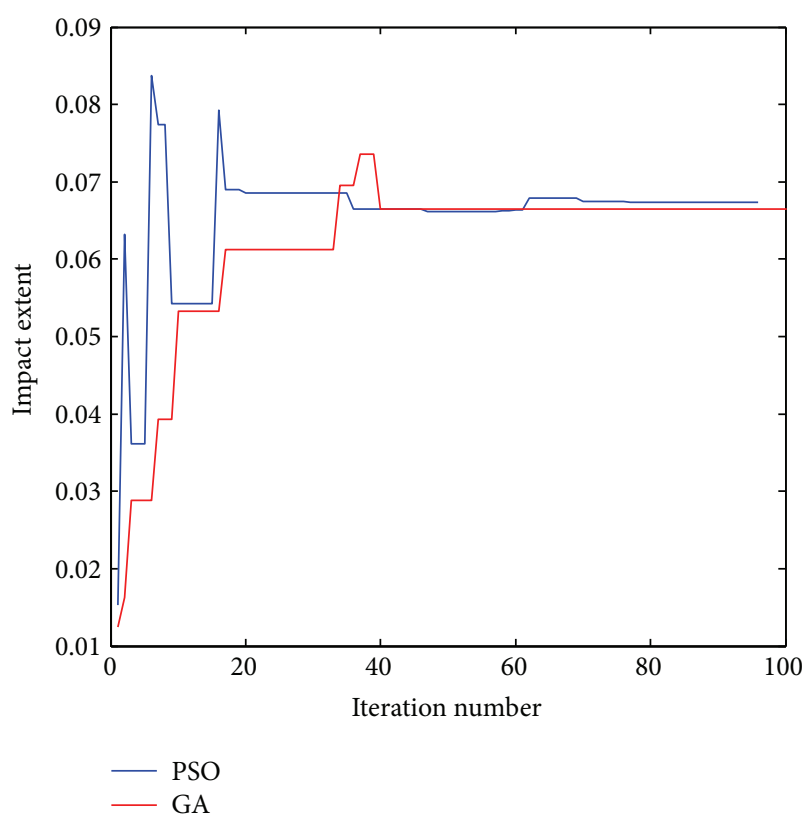

(b)

FIGURE 5: Noise level $v_{i}=5 \%$; evolution of impact zone characteristics. (a) Impact centre and (b) impact zone extent.

as a function of measurement noise level. It was found that PSO algorithm continues to achieve exact prediction of the solution even in the presence of $2 \%$ of noise intensity, while GA algorithm fails at that noise as the associated error was too large. Both of these algorithms fail to predict correct impact extent when noise level reaches $5 \%$, even if they continue to predict the position of the impact centre with rather acceptable accuracy.

To assess predictability in a large sense, system model noise should also be integrated in the future in conjunction with measurement noise.

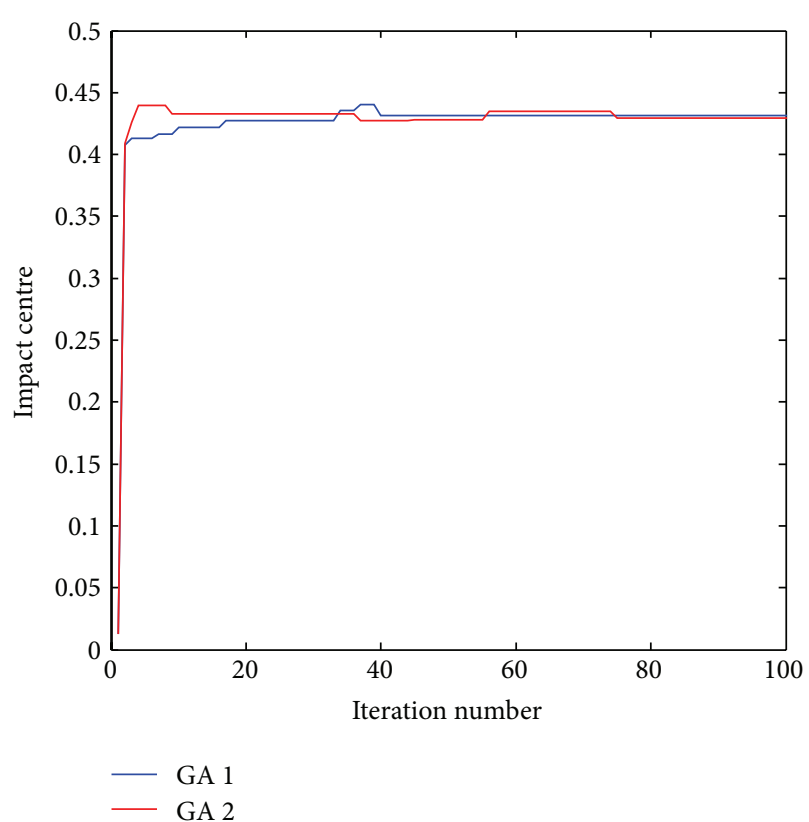

(a)

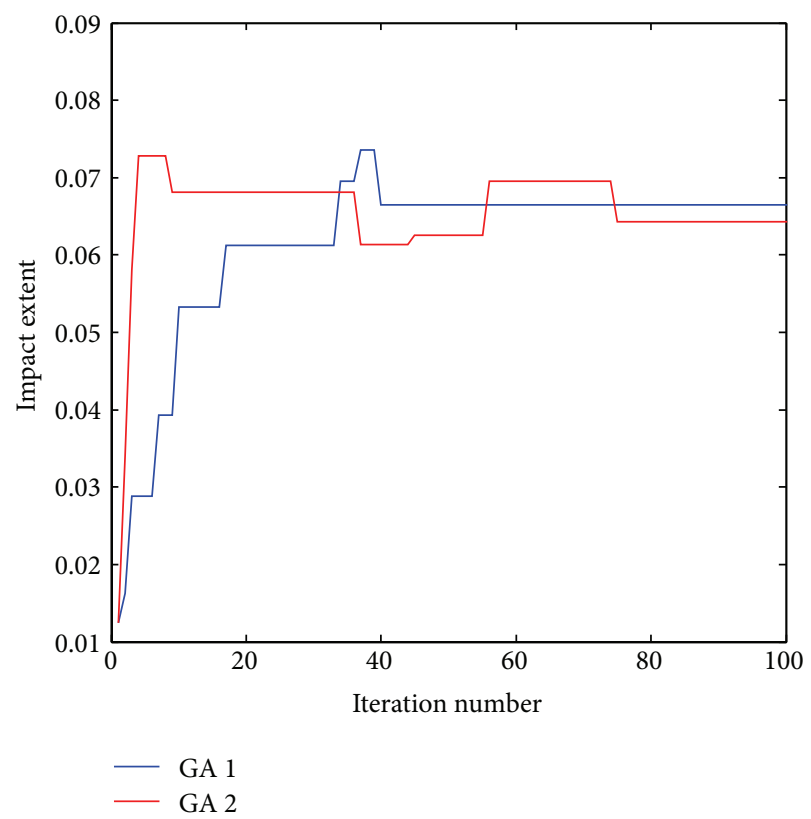

(b)

FIgURE 6: Noise level $v_{i}=5 \%$ for the four sensors; evolution of impact centre and extent as function of iterations (a) first test GA 1 and (b) second test GA 2.

\section{References}

[1] J. F. Doyle, "Experimentally determining the contact force during the transverse impact of an orthotropic plate," Journal of Sound and Vibration, vol. 118, no. 3, pp. 441-448, 1987.

[2] J. F. Doyle, "An experimental method for determining the dynamic contact law," Experimental Mechanics, vol. 24, no. 1, pp. $10-16,1984$.

[3] M. Tracy and F.-K. Chang, "Identifying impacts in composite plates with piezoelectric strain sensors-part I: theory," Journal 
of Intelligent Material Systems and Structures, vol. 9, no. 11, pp. 920-928, 1999.

[4] M. T. Martin and J. F. Doyle, "Impact force identification from wave propagation responses," International Journal of Impact Engineering, vol. 18, no. 1, pp. 65-77, 1996.

[5] H. Inoue, K. Kishimoto, and T. Shibuya, "Experimental wavelet analysis of flexural waves in beams," Experimental Mechanics, vol. 36, no. 3, pp. 212-217, 1996.

[6] C. S. Yen and E. Wu, "On the inverse problem of rectangular plates subjected to elastic impact-part 1: method development and numerical verification," Journal of Applied Mechanics, vol. 62, pp. 692-698, 1995.

[7] C. S. Yen and E. Wu, "On the inverse problem of rectangular plates subjected to elastic impact-part 2: experimental verification and further applications," Journal of Applied Mechanics, vol. 62, pp. 699-705, 1995.

[8] K. Choi and F.-K. Chang, "Identification of foreign object impact in structures using distributed sensors," Journal of Intelligent Material Systems and Structures, vol. 5, no. 6, pp. 864869, 1994.

[9] E.-S. Shin, "Real-time recovery of impact force based on finite element analysis," Computers and Structures, vol. 76, no. 5, pp. 621-635, 2000.

[10] J. F. Doyle, "A genetic algorithm for determining the location of structural impacts," Experimental Mechanics, vol. 34, no. 1, pp. 37-44, 1994.

[11] J. H. Holland, Adaptation in Natural and Artificial System, University of Michigan Press, Ann Arbor, Mich, USA, 1975.

[12] R. Eberhart and J. Kennedy, "New optimizer using particle swarm theory," in Proceedings of the 6th International Symposium on Micro Machine and Human Science, pp. 39-43, Nagoya, Japan, October 1995.

[13] J. Kennedy and R. Eberhart, "Particle swarm optimization," in Proceedings of the IEEE International Conference on Neural Networks, pp. 1942-1948, Piscataway, NJ, USA, December 1995.

[14] S. Koziel and Z. Michalewicz, "Evolutionary algorithms, homomorphous mappings, and constrained parameter optimization," Evolutionary Computation, vol. 7, no. 1, pp. 19-44, 1999.

[15] Y. Shi and R. Eberhart, "Modified particle swarm optimizer," in Proceedings of the IEEE International Conference on Evolutionary Computation (ICEC '98), pp. 69-73, May 1998.

[16] Q. He and L. Wang, "An effective co-evolutionary particle swarm optimization for constrained engineering design problems," Engineering Applications of Artificial Intelligence, vol. 20, no. 1, pp. 89-99, 2007. 

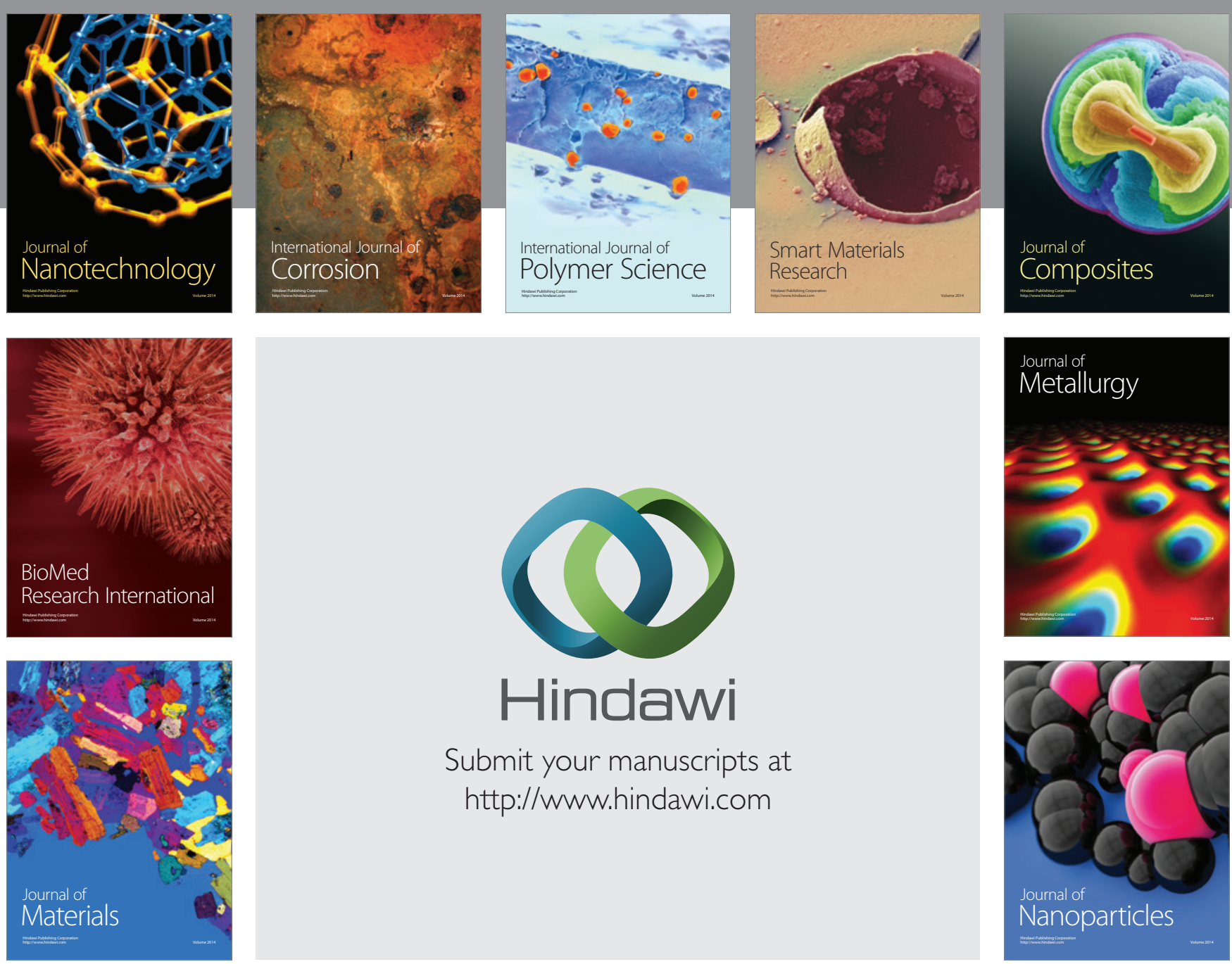

Submit your manuscripts at http://www.hindawi.com
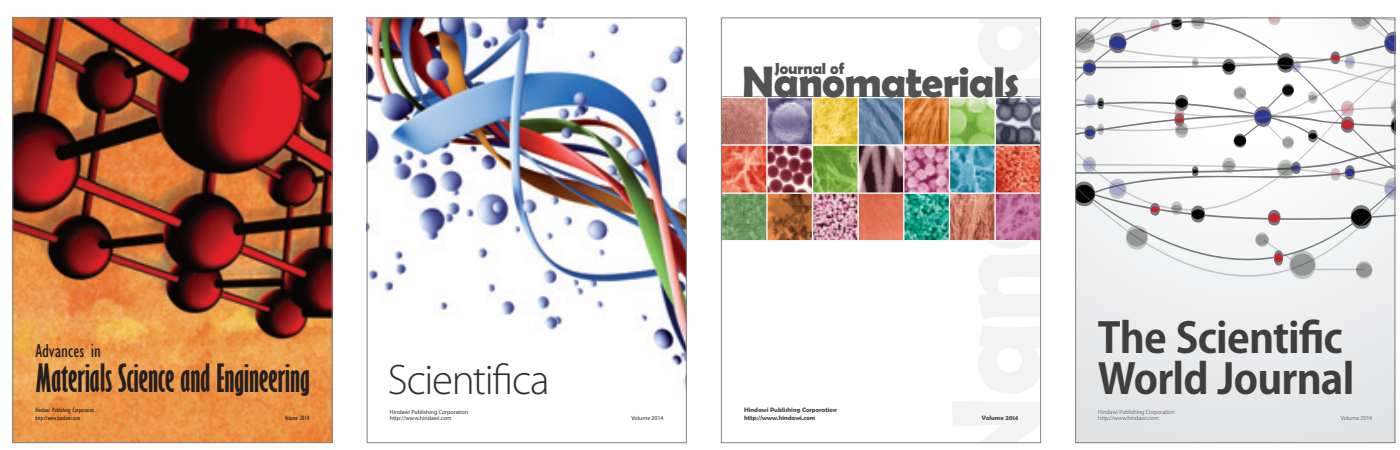

\section{The Scientific World Journal}
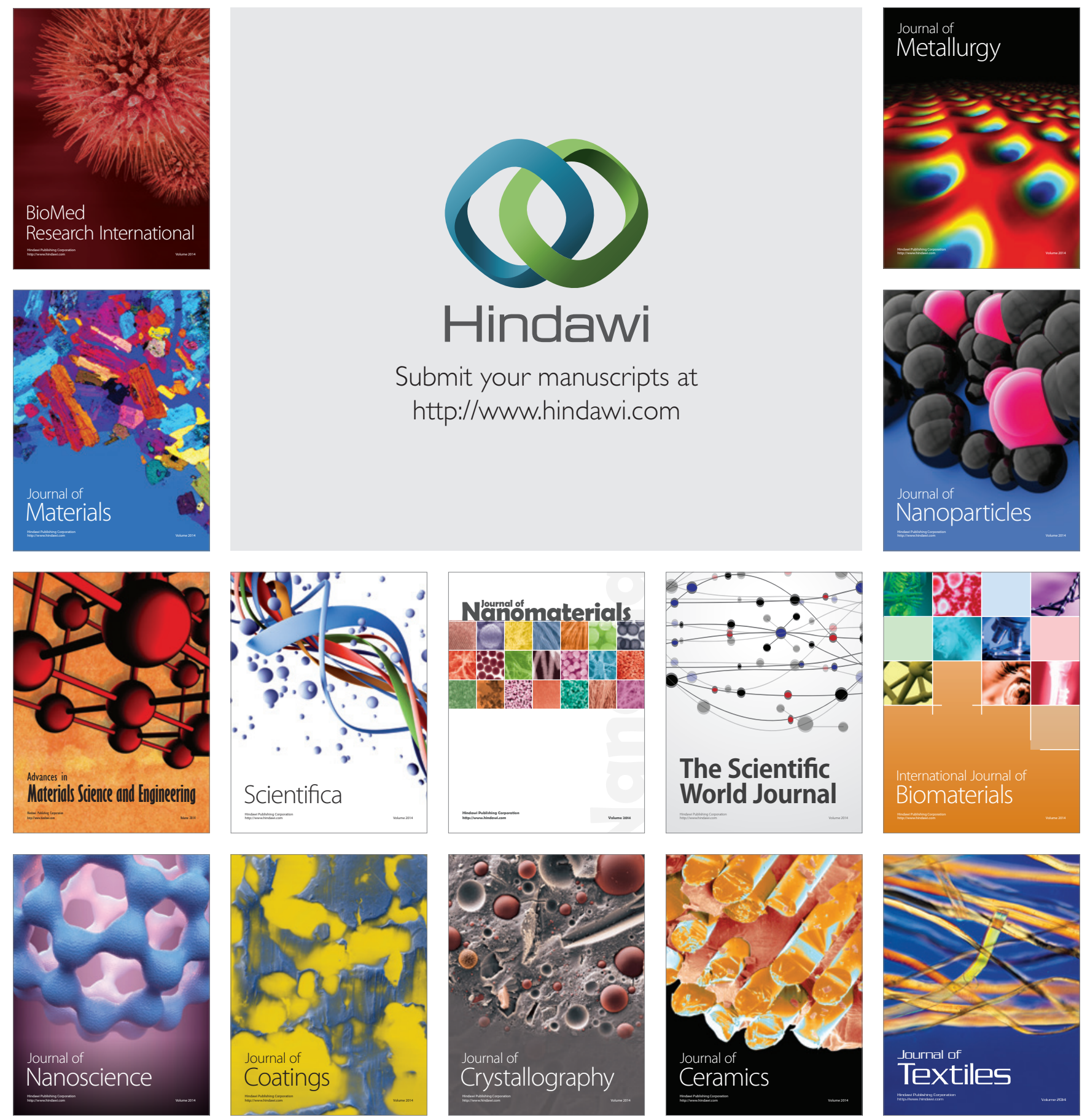